\title{
A full experimental and numerical modelling of the practicability of thin foam barrier as vibration reduction measure
}

\author{
M. Naghizadehrokni ${ }^{1}$, M. Ziegler ${ }^{1}$, and J. Sprengel ${ }^{2}$ \\ ${ }^{1}$ RWTH Aachen University, Department of Civil Engineering, \\ Chair of Geotechnical Engineering, Aachen, Germany \\ ${ }^{2}$ Vössing Engineer, Former GiB RWTH Aachen University
}

September 10, 2020

\begin{abstract}
This paper presents the performance of geofoam-filled trenches in mitigating of ground vibration transmissions by the means of a full experimental study. The results are interpreted in the frequency domain. Fully automated $2 D$ and $3 D$ numerical models are applied to evaluate the screening effectiveness of geofoam-filled trenches in the active and passive schemes. Experimental results are in good agreement with the prediction of numerical modelling. The validated model is used to investigate the influence of geometrical and dimensional features on the trench. In addition, three different systems including single, double and triangle wall obstacles are selected for analysis, and the results are compared for various situations. The parametric study is based on complete automation of the model through coupling finite element analysis software (Plaxis) and Python programming language to control input, change the parameters, as well as to produce output and calculate the efficiency of the barrier. The results show that the depth and the width of approximately $1 \lambda_{\mathrm{r}}$ and $0.2 \lambda_{\mathrm{r}}$, respectively are enough to reach the acceptable amount of efficiency for the active isolation for all three systems. For the passive scheme, the role of depth can be ignored for the single and double wall barriers, while depth plays a significant role for the triangle wall system.
\end{abstract}

\section{Introduction}

In metropolitan cities, man-made ground vibrations generated by external dynamic sources including traffic, blasting, machine foundations and other constructional activities have recently become a major concern for the nearby structures and inhabitants. Most of the transmitted vibration energy is propagated by Rayleigh waves close to the soil surface. The rest of the energy is transmitted by the body waves [1]. Near-surface vibration can be a major problem for the surrounding buildings and their inhabitants. Therefore, implementing a wave barrier in the transmission path is a suitable solution as it allows scattering 
the ground-borne vibrations 2. The barrier intercepts the dispatched waves through a complex mode of wave interactions including, reflection, refraction, diffraction and wave interference. Vibration screening is classified into two categories including the active scheme, in which the obstacle is installed near the source of vibration, and the passive scheme, in which the trench is constructed far away from the vibration source. On the other hand, the applicability of the wave barrier is classified in terms of open or in-filled trenches 3 .

Several studies including both field tests [4, 5] and numerical modelling [6] 8 have been carried out in order to evaluate the efficiency of the wave barrier in wave attenuation. Woods [9] was one of the first to perform a full experimental test for evaluating the efficiency of open trenches. He suggested that a wave barrier should reach at least $75 \%$ mitigation in screening of incoming waves to be deemed as efficacious. In practice, however, open trenches are not applicable in many cases for a longer time due to difficulties in construction and stability problems. In such cases, in-filled trench obstacles are generally used.

A series of field tests are conducted by [10, 11] for finding the screening effectiveness of open and geofoam-filled obstacles and found that the performance of geofoam-filled trench reached 68 and $67 \%$, respectively. Multiple rows of geofoam-filled barriers are employed for assessing the applicability of trench in frequency domain [12. They proved that increasing trench depth, to more than one wavelength has only negligible affect on screening performance in the passive scheme. Celebi et al. [13] described the results of experimental studies to compute the screening efficiency of open, stiff and soft barriers in the case of active and passive schemes. The observations illustrated that the passive isolation is more effective than the active isolation and softer back-fill material can be more effective in attenuating the incoming waves in comparison with the stiff materials. In addition, a centrifuge parametric investigation is accomplished by 14 to examine the effect of dimensional and geometrical factors on the geofoam-filled trench in the mitigation of incident waves. The results of the study showed that the screening performance is dependent on the trench depth. In addition, numerical techniques including the finite element method (FEM) and the boundary element method (BEM) can be used as powerful tools to study the screening performance of wave barriers. Beskos et al [15 17] developed a BEM model to determine the screening capability of open and concretefilled trenches in homogeneous and layered soil. Ahmad and Al-Hussaini 18 conducted an extensive parametric study to analyse the influence of different geometrical and material parameters on the screening efficiency of rectangular open and concrete-filled obstacles. Finally, they developed a simple model for vibration isolation in active and passive schemes. Haupt [19, 20] also used numerical modelling for assessing the vibration isolation performance. He suggested that the trench depth should be at least $80 \%$ of the R-wavelength $\left(\lambda_{\mathrm{r}}\right)$ to reach a successful attenuation. A FEM is developed to evaluate the efficiency of water and geofoam-filled trenches [21, 22. They observed that the depth of the barrier is the governing factor in increasing the efficiency of the barrier and width and length of the trench do not play a significant role in screening performance. Majumder et al. 23 25 performed some numerical modelling in order to evaluate the efficiency of the geofoam-filled trench. They suggested the normalized depth equal to 1 as the optimum value for reaching the highest value of the efficiency. In addition, they stated that increasing the frequency of the vibration source results in increasing the efficiency of the trench. Qiu et al. 26] 
established a $2 D$ numerical model for a comparative study on vibration isolation effectiveness and developed a model for optimizing various dimensions of the barrier. Concerning different configurations of the barriers, Saikia [27] and Schevenels et al. 28] considered double wall barriers, Esmaeili et al. [29, 30] evaluated the effectiveness of $\mathrm{V}$-shaped and step-shaped trenches and Alzawi et al. 31] conducted a comprehensive study for computing the performance of geofoam-filled barriers in the form of box-walls, single and double wall systems. Studying the literature revealed that a full experimental test and a parametric investigation to examine the efficiency of various configurations of geofoam-filled trenches are required. This paper deals with filling this gap through a field test and a comprehensive parametric study.

The results of the field tests for evaluating the efficiency of geofoam-filled barrier in mitigating the incident waves in the frequency domain are presented in section 2, $2 D$ and a $3 D$ finite element models are developed and the validity of the models are compared with the results of the full experimental test as shown in section 3.1. Subsequently, different configurations including single, double and triangle wall systems are selected for evaluating the performance of vibration screening in the active and passive schemes in sections 4 and 5 , respectively. Various parameters including geometrical parameters and dimensional factors that govern the screening performance are considered for more assessing.

\section{Field Test}

\subsection{Site Investigation}

A full experimental test has conducted 32 to analyse the efficiency of the geofoam-filled trench in screening dispatched waves. The subsoil properties and the location of the field test are one the most important features. The main criteria for selecting the site location are considered as homogeneity of the soil, easy accessibility to the test site and possibility of the implementation of the trench. For these purposes, the location of the test site has selected in a gravel pit based on a flat area with dimensions of approximately $40 \mathrm{~m} \times 60 \mathrm{~m}$, located in Geilenkirchen, a town in North Rhine-Westphalia.

The soil analysis consisted of soil classification and has done on some samples from different depths. The result of laboratory test of the soil demonstrated that the subsoil is a mixture of sand and gravel up to a depth of at least $15 \mathrm{~m}$. According to DIN 18196, the soil is classified as intermittent graded sand. The medium soil density was determined to $\left(\rho=2 \mathrm{~g} / \mathrm{cm}^{3}\right)$.

The Multichannel Analysis of Surface Waves method (MASW) was used to determine the dynamic elastic material parameters, which are key factors for describing the soil dynamic behaviour of the subsoil. MASW is a geophysical procedure for determining the depth-dependent profile of the propagated shear wave velocity. The data were recorded over a total of 48 geophones, which were laid out at a distance of $1 \mathrm{~m}$, and registered by a seismograph. Fig. 1 shows the geophones in the field test and the determined profile of the propagation velocity of the shear wave over the depth as an average value over the entire measuring section. 


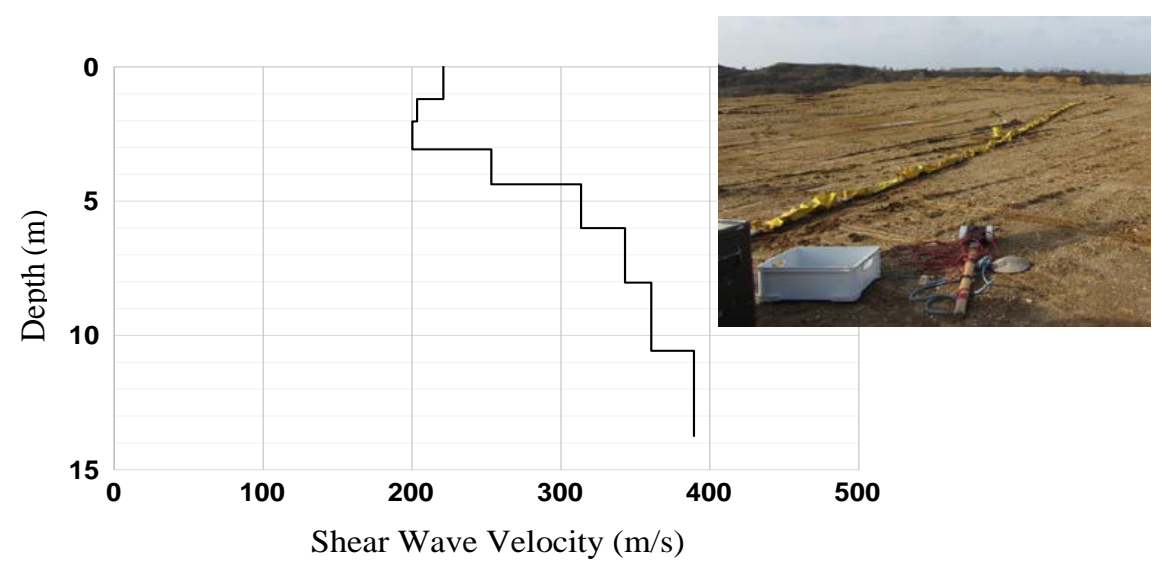

Figure 1: Shear wave velocity profile of the site

\subsection{Field Test Method and Equipment}

A shaker from Baudynamik Heiland \& Mistler GmbH was used (see Fig. 2A), which allows to produce a harmonic excitation of the ground [33. The shaker was founded on four steel plates and the overall dimensions of the shaker were $72 * 72 \mathrm{~cm}$. The servo-hydraulic shaker simulated the requested objects, in this case, harmonic load in the frequency range of theoretically 0.01 to $110 \mathrm{~Hz}$ with $\bullet$ The maximum force amplitude is about $20 \mathrm{kN}$, but the dynamic force amplitude is about $15 k N$. The force amplitude generated in the field test was constantly $5 \mathrm{kN}$ in the frequency range from $10 \mathrm{~Hz}$ to $110 \mathrm{~Hz}$, but only the range from $20 \mathrm{~Hz}$ to $80 \mathrm{~Hz}$ was used for trench efficiency evaluation [32. The measurement of the
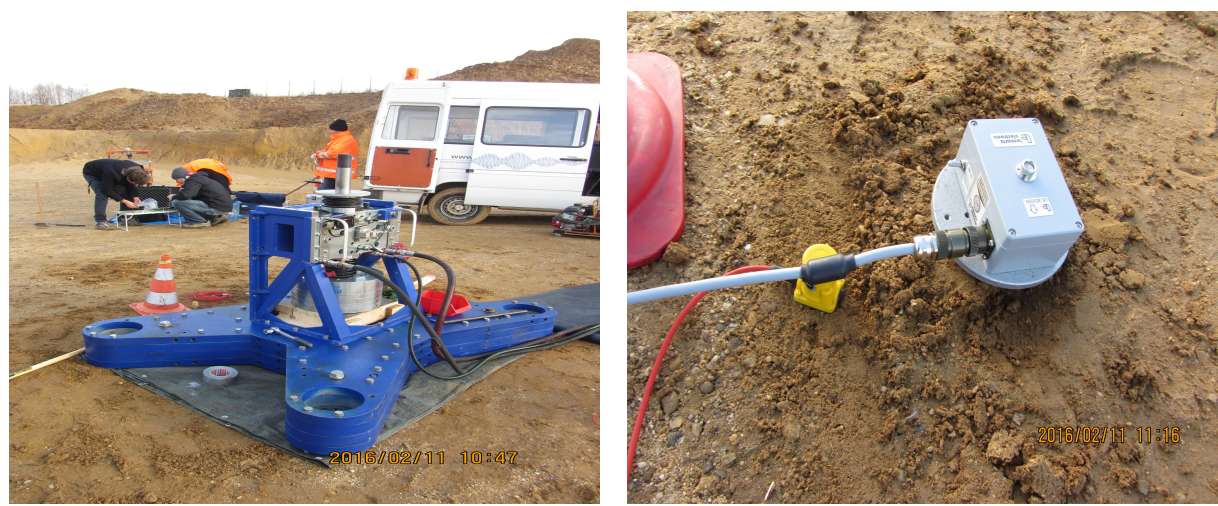

Figure 2: Vibration source: (A) Shaker structural dynamics Heiland \& Mistler GmbH, (B) Vibration sensor

vertical soil particle velocity had been done two times, before installation the trench and one after implementation of the geofoam-filled barrier. The measuring chain in the field test consisted of the vibration sensors including geophone and accelerometer shown in Fig. $2 B$, which transmitted their data to a measuring computer. The transferred data were analysed by MESSI, a program developed by the Department of Structural Mechanics at RWTH Aachen Uni- 
versity for signal acquisition and processing. Some distinct points were selected for installing vibration sensors with different distances from the source of vibration and the obstacle. Fig. 3 illustrates the selected measuring arrangement including the positioning of the vibration source, the geofoam-filled barrier and the vibration sensors, respectively. The shaker was located at two different dis-

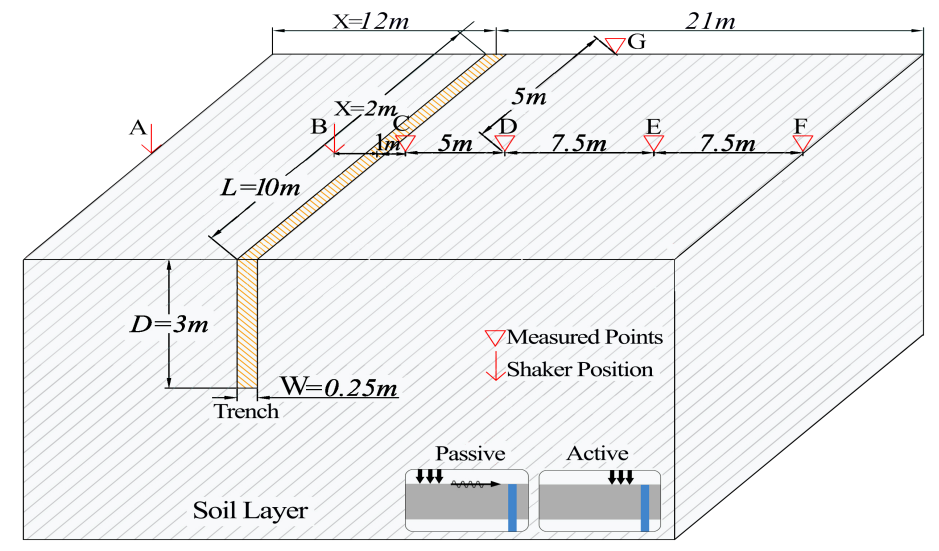

Figure 3: Measuring arrangement

tances from the trench. To investigate the effectiveness of the obstacle for both situations, the vibration source had appropriately positioned relative to the barrier. Due to the limited number of available vibration sensors, seven measuring points were selected per measurement series, with measuring points $\mathrm{A}$ and $\mathrm{B}$ located directly on the shaker for the passive and active isolation, respectively. An approximate propagation velocity of the R-wavelength at the surface of $v_{r}=200 \mathrm{~m} / \mathrm{s}$ and a minimum and maximum examined frequency of 20 and $100 \mathrm{~Hz}$ leads to a wavelength of $\lambda_{\mathrm{r}}=10$ and $2 \mathrm{~m}$. Therefore, the position of vibration source was chosen $2 m$ for the active isolation and $12 m$ for the passive isolation, respectively. Excavating a trench for mitigating of incident waves was the next step after measuring the vertical soil particle velocity in the absence of the barrier. Most of previous studies [8, 15, 34] stated that a normalized depth of $0.8 \lambda_{r}$ is enough to reach acceptable value of the efficiency. Therefore, an attempt was made to reach this depth during the experimental test. A wheel trencher device was used for digging the trench with the dimensions of $3.2 \mathrm{~m}$ depth, $20 \mathrm{~cm}$ width and $10 \mathrm{~m}$ length. However, the excavating process to reach this depth failed because of facing hard soil in some sections. Therefore, the excavated depth of the barrier was between $2.8-3.2 \mathrm{~m}$ and the average depth of $3 \mathrm{~m}$ was considered for whole trench. The low stability of the trench due to the kind of soil resulted in a very short stability of the barrier. Therefore, the foam was injected immediately after excavating the trench. The device during digging the trench and final geometry of the injection body is shown in Fig. 4. The geofoam material for filling the barrier was a PU foam from Uretek Deutschland $\mathrm{GmbH}$ that could be injected in the trench without any resistance and expanded freely; all these factors lead to a geofoam with very low density. 
A laboratory test was carried out for finding the dynamic properties of the geofoam. The results showed a medium density of $0.61 \mathrm{kN} / \mathrm{m}^{3}$, shear wave velocity of $330 \mathrm{~m} / \mathrm{s}$ and poison's ratio close to zero $(0.01)$.
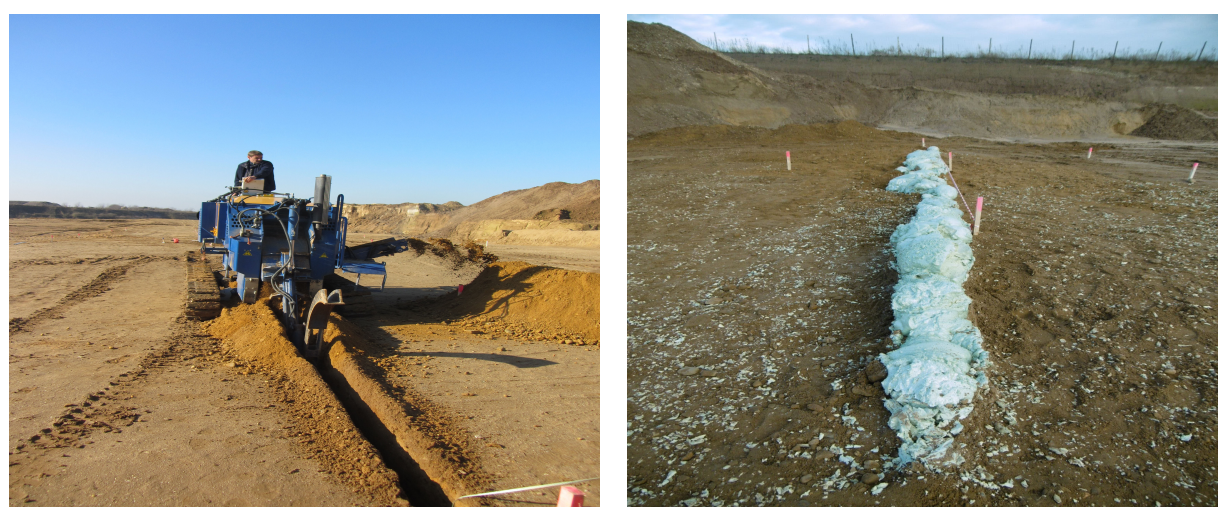

Figure 4: Wheel trench device and injection of geofoam

\subsection{Test Results and Discussion}

The test results of the field-test are evaluated in terms of the vertical vibration velocities of the soil, which are measured in the time domain and transmitted into the frequency domain by applying Discrete Fourier Transformation (DFT). The results are presented in the frequency domain. An advantage of representing the results in the frequency domain is to visualize the wave interference influences. The final assessment of the effectiveness of the trench is made by comparing the two measurements.

Result of full experimental study for all measured points beyond the geofoamfilled trench are analysed and the results are presented in following figures. In order to ensure reliability of the results, the measurements have been done two times for both before and after installing the barrier. Fig. 5 shows a significant attenuation for almost all measured points for the frequency between 30 and $60 \mathrm{~Hz}$ by implementing the geofoam-filled trench. The results shows the highest amount of mitigation for the first point after the trench (point C) in comparison with other points. As the frequency of applied load increases, the vertical soil velocity amplitude reduces dramatically and then gradually reaches to a stable value after a frequency of $60 \mathrm{~Hz}$. The system effectiveness is increased from 37 to $60 \%$ by increasing the frequency from 30 to $40 \mathrm{~Hz}$ for point C. A practical comparison can be done between the screening of incident waves for measured points of $\mathrm{D}$ and $\mathrm{G}$, where are located at the center and beside of the source in Fig. 3. respectively. The results reveal that we have the highest amount of mitigation in the centreline of the trench in comparison with other areas. The efficiency of the frequency of $40 \mathrm{~Hz}$ for both points D and $\mathrm{G}$ are 65 and $35 \%$, respectively, which shows a considerable difference.

However, there is not any attenuation effect for the frequency less than $20 \mathrm{~Hz}$ and it seems that the isolation system is not practical for lower frequencies. Athanasopoulos et al. 35 stated that the attenuation of $\mathrm{R}$-wave amplitude is frequency-dependent and it increases exponentially with the frequency due to both radiation and material damping. Therefore, low frequency of the load 

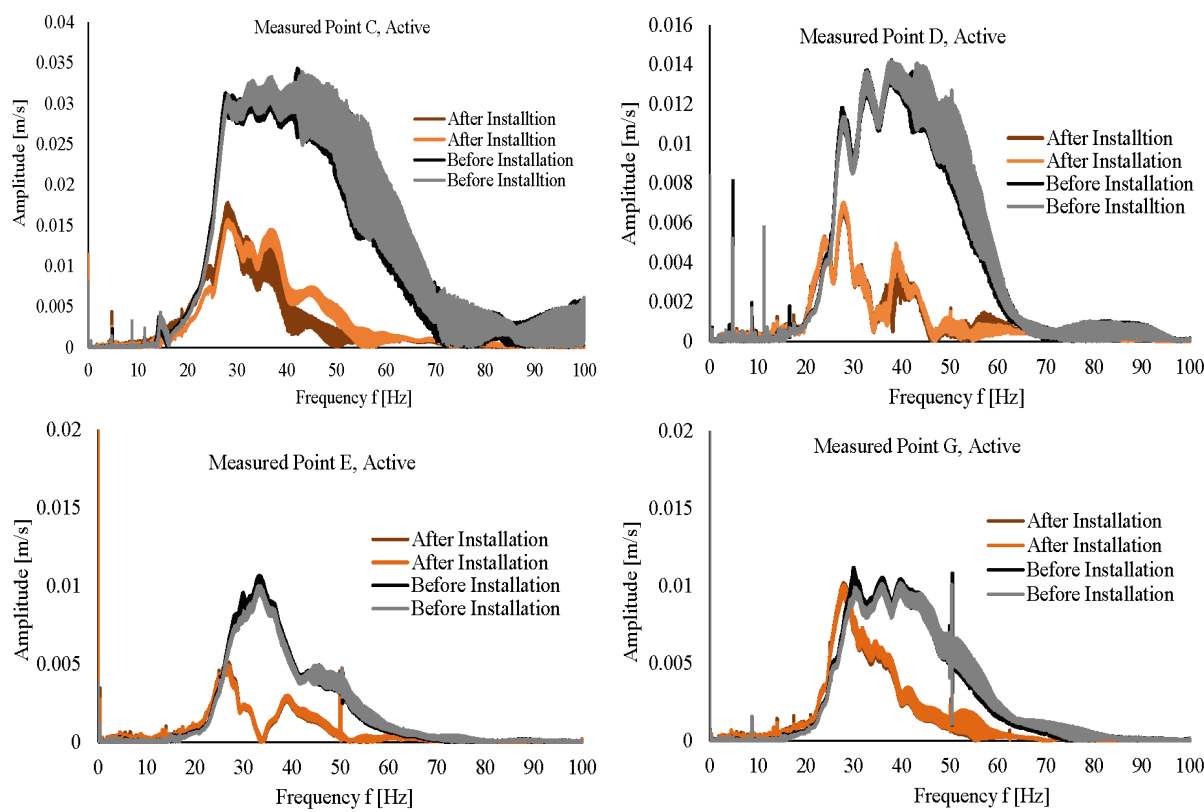

Figure 5: Representation in the frequency domain: Measuring points C, D, E, $\mathrm{G}$ (Active Isolation)

results in less attenuation of the propagated waves in the soil. This issue disappears the effectiveness of the trench in mitigating the waves with large Rwavelength (which is equal to low frequency). The same statement can be applied for the reason of not practicability of the trench in high frequencies. Increasing the frequencies result in losing of the wave energy until disappearing. Therefore, there is not any difference between amplitude of the vertical soil particle velocity before and after installing the trench. The results of various points for the passive isolation are presented in Fig. 6. The area immediately after the trench has the highest screening performance. The screening performance in attenuation of incident waves increases for the frequency range between 30 to $60 \mathrm{~Hz}$, whereas this frequency range decreased to $35-50 \mathrm{~Hz}$ for point E. However, some unexpected peaks and valleys are observed in vertical soil velocity amplitude of 50 and $40 \mathrm{~Hz}$ for points $\mathrm{C}$ and $\mathrm{D}$, respectively.

Baidya, et all [36 investigated change of amplitudes of vibrations in layered soil and they stated that many soils in nature exhibit an increase in shear modulus with depth and this issue can be recognized as a reason for distinct peaks and valleys. The incoming waves may reflect and refract at the interfaces of soil layer and they can superpose with surface waves in the same phase and originate a bigger wave, which cause amplification in vertical response of the soil. As it is already mentioned in section 2.2 , we faced a hard soil during the excavation and for this reason the depth of the trench could not reach the target value $(3.2 m)$. It means that we have almost a full reflection of incoming waves. Therefore, the unexpected discrepancies in the result can be explained by superposition of surface waves and reflected waves 10 . In addition, Haupt stated that the reflection and refraction of the R-wave energy generates a typical interference pattern, in which the maximum or minimum points usually appear at intervals 
of half of R-wave [19]. By following the intervals for each minimum/maximum point in the figure, it can be seen that the intervals follow the rules of Haupt. This pattern is generated by the superposition of waves.
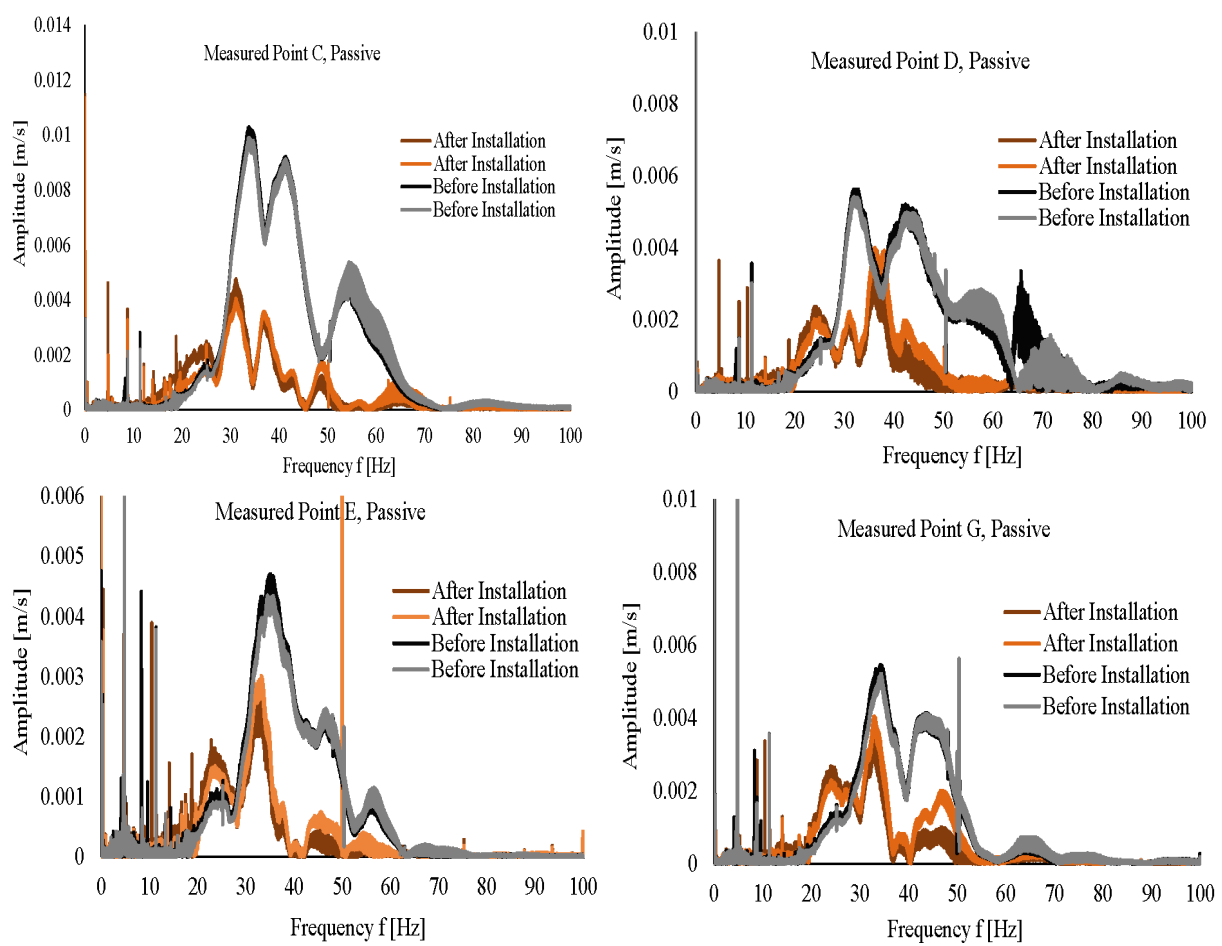

Figure 6: Representation in the frequency domain: Measuring points C, D, E, $\mathrm{G}$ (Passive Isolation)

Frequency domain analysis of the results enables to monitor normalized trench depth in terms of R-wavelength. Changing the frequency of the load leads to changing the length of $\mathrm{R}$-wave since the shear wave velocity of the soil is constant. Therefore, the effectiveness of the geofoam-filled trench is presented as a normalized depth in Fig. 7. The results show that increasing $D$ results in decreasing the value of $\left(\bar{A}_{r}\right)$, which means increasing the efficiency of the trench. The lowest value of $\left(\bar{A}_{r}\right)$ is achieved for a normalized depth of approximately 0.6 , which agrees with the result of [1].

Average amplitude reduction ratio is expected to decrease with increasing the depth of the trench. However, some amplifications in $\left(\bar{A}_{r}\right)$ are observed after the normalized depth of 0.6. Haupt stated that distinct peak can occur when shear wave velocity of the soil increases proportional with the depth. In addition, Massarsch [4] indicated that refracted waves from the trench and transferred waves beneath the trench can superpose in the same phase and originate a bigger wave, which can cause amplification. Since the soil profile in experimental test is non-homogeneous in terms of increasing shear wave velocity, the unexpected distinct peaks can be explained by superposition of surface waves. 


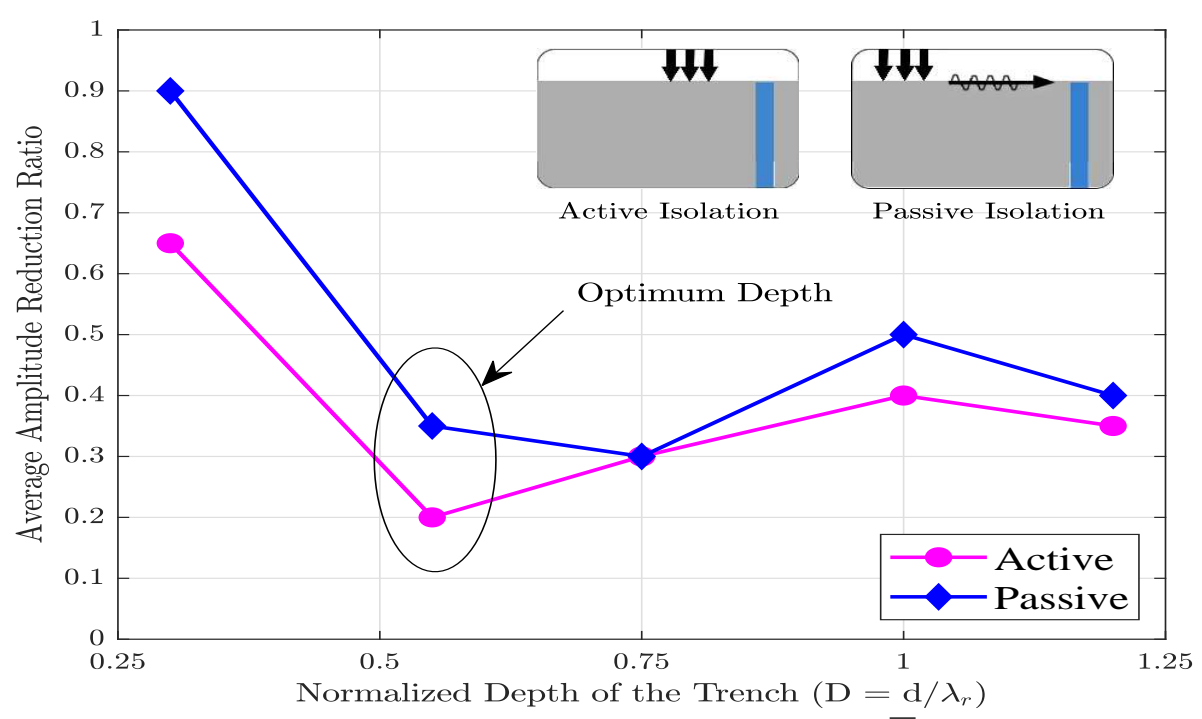

Figure 7: Influence of normalized barrier depth on $\bar{A}_{r}(X=0.5)$

\section{Numerical Modelling}

Since the model is largely affected by the transmitted and reflected waves from the boundary of the model, it is important to keep the region of interest enough far from the reflecting boundary. Therefore, the dimensions of the model are selected as $(50,20,20 \mathrm{~m})$ and $(70,25 \mathrm{~m})$ in the case of active and passive system, respectively. The soil is modelled as a homogeneous and isotropic half space. The linear elastic model is selected for the soil since the amplitude of generated vibrations are small and the soil does not experience the development of plastic deformations in the region of consideration. In order to applying a special boundary condition, viscous boundaries are applied to the bottom and right sides of the $2 D$ and $3 D$ models and at $Y$ max direction for $3 D$ model, too. A viscous boundary condition, which was introduced by Lysmer and Kuhlmeyer [37. consist of the viscous dampers applied in $X$, and $Y$ (for $2 D$ ), and $Z$ (for $3 D$ ) direction along the boundary. Relaxation coefficients $(C 1$ and $C 2)$ are introduced in order to improve the effect of absorption. Improving the wave absorption in the normal and tangential direction is done by coefficients $C 1$ and $C 2$, respectively. The values for both coefficients are selected as 1 .

Plaxis uses a global meshing parameter, which is called average element size for showing the quality of the mesh. This parameter is calculated based on the dimensions of the model and the number of elements. The average element size $\left(I_{e}\right)$ of 0.5 is enough for reaching acceptable results and satisfying the criteria of Kuhlemeyer and Lysmer [37] for assuming the average element size less than to one-eight of the wavelength associated to the maximum frequency component of the input wave. The vibration source is modelled as a vertical harmonic load with the amplitude of 1 and frequency of $50 \mathrm{~Hz}$. The dynamic time interval is considered as $0.5 \mathrm{~s}$, which is enough to allow the passage of dynamic load. The steel plate under the shaker is modelled as plate and the shaker weight is considered as a uniform distributed load with magnitude of $1 \mathrm{kN} / \mathrm{m}^{2}$. However, weight of the steel plate and the shaker is kept constant during parametric study since 
it does not play a significant role in vibration attenuation results and the difference for the efficiency of trench between zero and non-zero foundation weight is $1.5 \%$, which can be neglected [11, 34, 38.

A large number of models are developed for the parametric study through changing the governing factors of the trench. Creating all models manually is a timeconsuming process. In order to save the calculation costs, a script is developed for coupling the finite element model (Plaxis) with Python language. This script allows the user to control both the input and the output of the model via an external Python handler. The main advantage of the automation is accelerating the pace of the parametric study. The input of the script corresponds to the values selected for different parameters, including the depth $D$, width $W$, length $L$, distance between the walls $S$ and the location $X$. Three different systems are selected including the single, double and triangle wall for assessing the effect of the shape of the trench on the screening performance. The script starts by creating a borehole and subsequently installs the trench, defines different phases, runs the model and calculates the efficiency of the trench. The entire process is executed automatically as shown in Fig. 8. Different functions are defined for excavating the trench with different configurations including the single, double and the triangular wall. An average amplitude reduction ratio factor $\left(\bar{A}_{r}\right)$ is used for finding the efficiency of the trench beyond the trench. The value of $\left(\bar{A}_{r}\right)$ is the average value of vertical soil velocity before and after the installation of the trench. Finally, the data for each system are stored in an Excel file. The created Python script for $2 D$ and $3 D$ model are provided in the Appendix.

The soil properties for the following analysis are considered the same as those measured in the field test, which are presented in table 11. The constant frequency of $f=50 \mathrm{~Hz}$ is selected for the applied load, which results in $\mathrm{R}$-wavelength equal to $4 \mathrm{~m}$. In addition, the linear elastic behaviour is used for both the geofoam and soil in the further calculations. The parameters $\alpha$ and $\beta$ are Rayleigh coefficients that determine the effect of mass and stiffness in the damping of system, respectively. In mass proportional damping, damping forces are generated by velocities of the model and simulated the model's movement through a still viscous fluid. Therefore, motion at points within the model cause damping. It should also be observed that the mass proportional damping would give damping due to rigid body motions. In stiffness proportional damping, damping is generated by strain rates. Therefore, the damping is more related to the material in comparison with motion of the model. Hence, stiffness proportional damping is used in this finite element model [14. In addition, in many practical soil related problems, $\alpha$ is ignored since it can lead to undesirable result if a large mass has been introduced into the model [39 41].

Table 1: material properties of Proposed FE model

\begin{tabular}{|c|c|c|c|c|c|}
\hline & $\begin{array}{c}\gamma \\
\left(k N / m^{3}\right)\end{array}$ & $\begin{array}{c}\text { Poisson's } \\
\text { ratio }\end{array}$ & $\begin{array}{c}\text { Shear wave } \\
\text { velocity }(\mathrm{m} / \mathrm{s})\end{array}$ & $\begin{array}{c}\text { Elastic Modulus } \\
\left(\mathrm{kN} / \mathrm{m}^{2}\right)\end{array}$ & $\begin{array}{c}\text { Rayleigh damping } \\
\text { coefficient }(\beta)\end{array}$ \\
\hline Soil & 20 & 0.3 & 216 & 250000 & 0.0003183 \\
\hline Geofoam & 0.61 & 0.01 & 330 & 13750 & - \\
\hline
\end{tabular}


5

stement

3

Create the

soil

stratigraphy

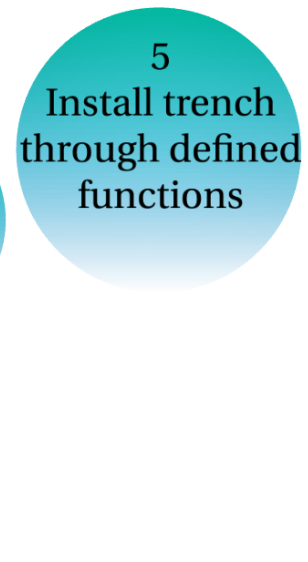

Plaxis Automation

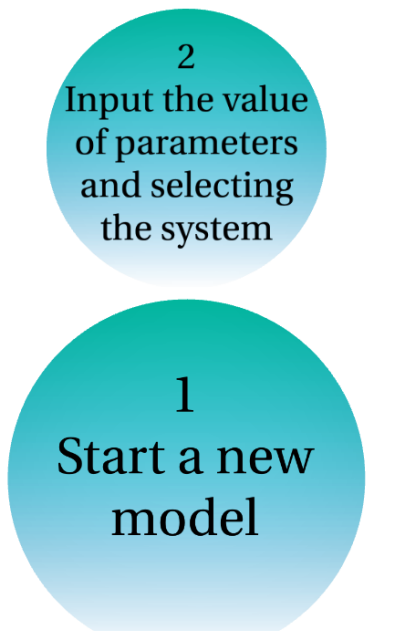

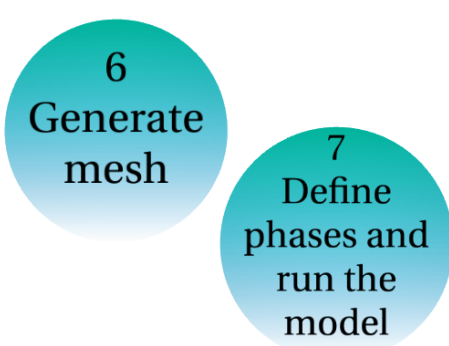

Collect results

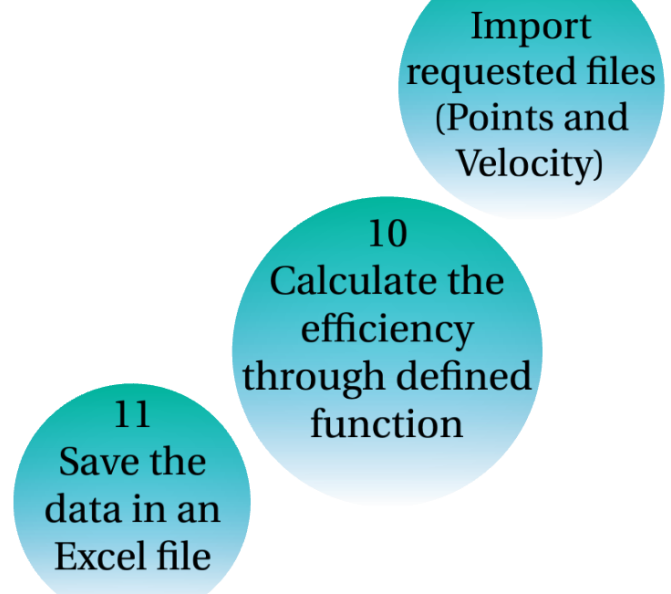

Figure 8: Automation process of Plaxis model with Python 


\subsection{Experimental Validation of Finite Element Method}

The developed model has been validated using the results of the field test for the active and passive isolations. The measured and the calculated wave attenuation curves are depicted in Figs. 9 and 10 using a logarithmic scale for different excitation frequency including, 20, 35, 50 and $65 \mathrm{~Hz}$, respectively.
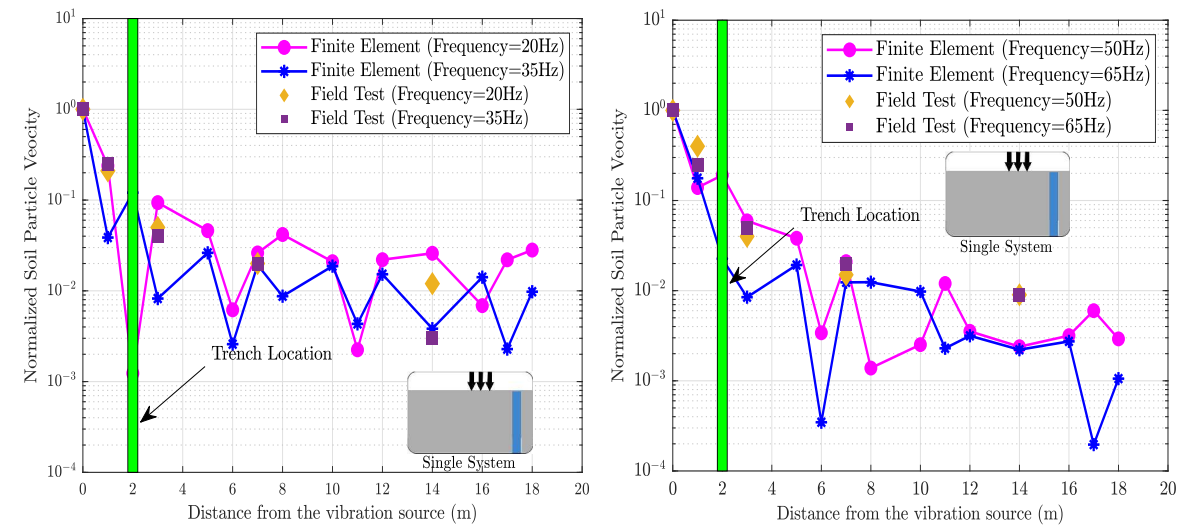

Figure 9: Validation of numerical modelling based on the result of field test, active isolation, $(\mathrm{D}=3 \mathrm{~m}, \mathrm{~W}=0.25 \mathrm{~m}, \mathrm{~L}=10 \mathrm{~m}, \mathrm{X}=2 \mathrm{~m})$
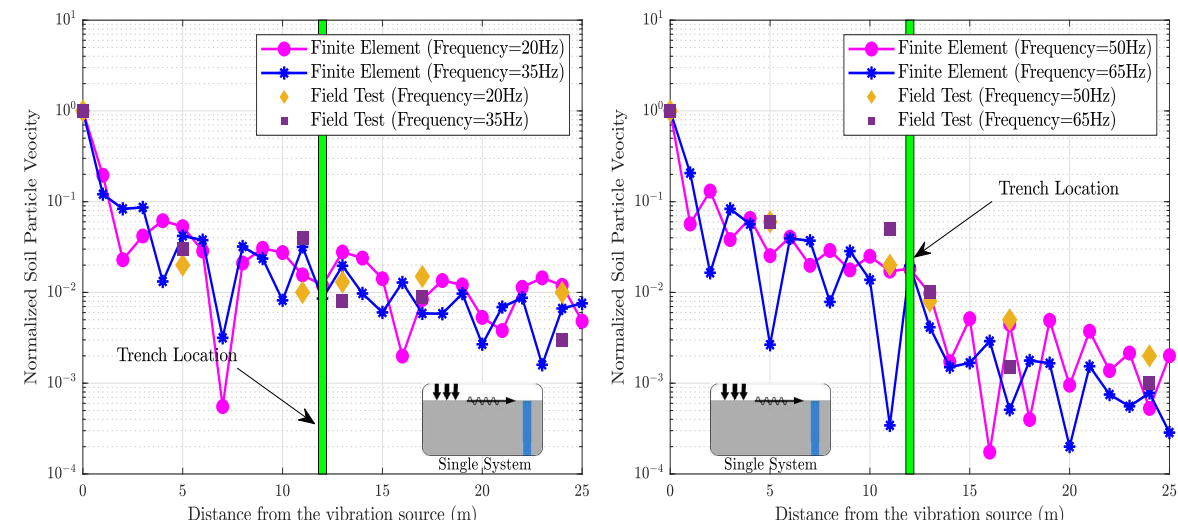

Figure 10: Validation of numerical modelling based on the result of field test, passive isolation $(\mathrm{D}=3 \mathrm{~m}, \mathrm{~W}=0.25 \mathrm{~m}, \mathrm{~L}=10 \mathrm{~m}, \mathrm{X}=12 \mathrm{~m})$

The FEM results are in line with the trends in the experimental results and there is a reasonable agreement between the normalized vertical soil particle velocities, obtained for both the active and passive cases. Nonetheless, unexpected valleys are visible in the results. Propagated waves through and underneath the geofoam-filled trench are sometimes in or out of phase. This results in a destructive interference of waves and creates valleys in numerical modelling results [21]. Another reason of observing some irregular peaks in the results is the less normalized distance between the points for presenting the numerical modelling, which is 0.25 . This distance results in presenting the results as detailed as possible and showing all irregularities that may happen for the area after the trench. The same figure has been illustrated with normalized distance of 0.5 
and fewer irregularities have been observed with new distance but the results of this distance is not presented here.

Therefore, the model developed in this study, including the wave absorbing boundary, adequately predicts the vibration propagation problem for the case of the geofoam-filled trench.

\section{Active Isolation}

A comprehensive parametric investigation is carried out to evaluate the effectiveness of different isolation systems including the single, double and triangular walls as the wave barriers for the active isolation due to the harmonic load in the vertical direction. Results of the detailed parametric investigation are presented and some applicable recommendations are suggested for designing the wave barriers.

The parametric study begins with considering the different normalized values with respect to the $\mathrm{R}$-wavelength for parameters of the trench including the depth $D$ (from 0.5 to 2.5 ), the width W (from 0.0625 to 0.25 ) and the length L (from 0.625 to 5 ), respectively. All parameters are normalized with respect to the $\mathrm{R}$-wavelength. The different geometrical parameters are considered to be the same for all configurations in this paper. Three different normalized locations including $0.5,1.25$ and 2 are selected for computing the efficiency of the geofoam-filled trench. Since the efficiency of the barrier for all three systems is not affected by the location of the trench, the result of one location (0.5) is presented.

\subsection{Single Wall System}

The normalized soil particle velocity before and after installing the trench was compared for an area beyond trench. The geofoam-filled trench was installed at the normalized location of 0.5. The results, which are not presented here showed that there is not any considerable difference after distance of $7.5 \lambda_{\mathrm{r}}$ for both with and without trench for the area beyond the barrier. This leads to use $7 \lambda_{\mathrm{r}}$ for the screened zone for calculating $\bar{A}_{r}$. This value is selected for all systems in the active scheme.

\subsubsection{Effect of Trench Dimensions}

Fig. 11 shows that $\bar{A}_{r}$ decreases dramatically, which is the result of increasing the normalized length from 0.625 to approximately 2.5 . It is apparent from the figure that the normalized length beyond around 2.5 does not create any changes and may be conservatively assumed to remind unchanged thereafter. The same parametric study has been applied to other systems with the same results. Therefore, the normalized length of the trench is considered as 2.5 for other systems as well. The influence of varying the depth of the EPS Geofoam wave barrier is studied and the results are presented in Fig. 12. We achieve a considerable amount of wave attenuation by increasing the depth of the barrier from 0.5 to 1 for all widths. A gradual increase or decrease in wave attenuation is observed when increasing the normalized depth up to 1 . It is observable that 


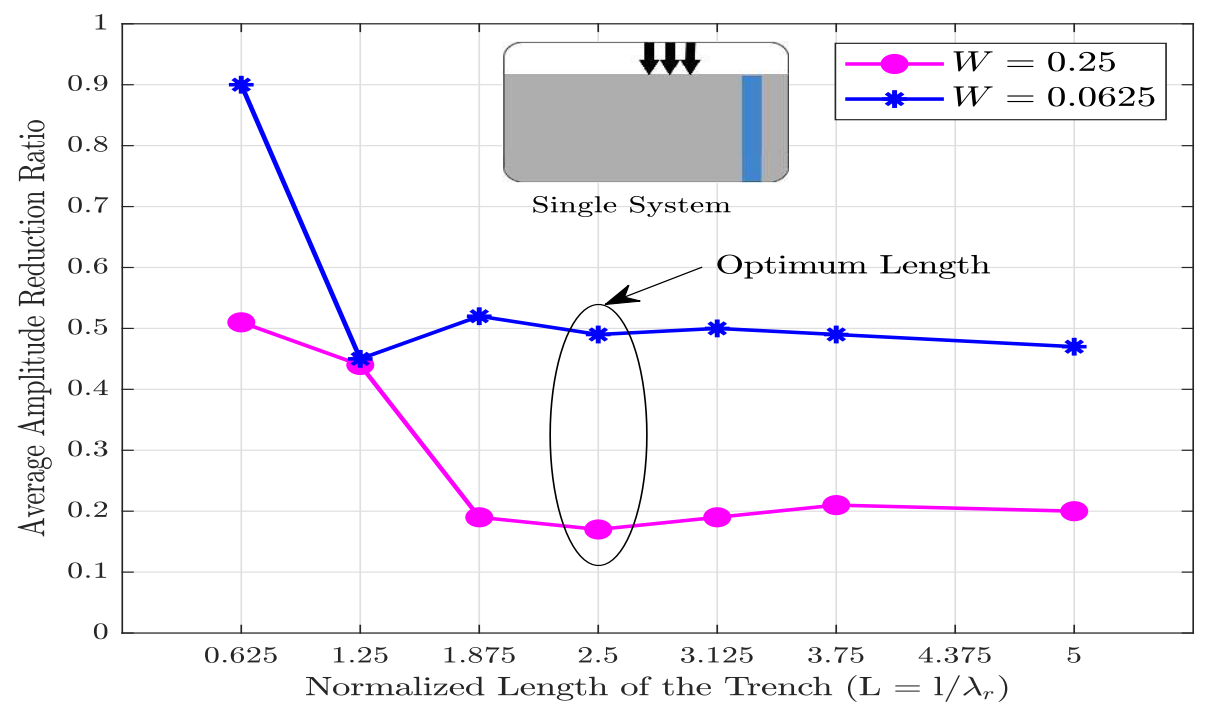

Figure 11: Influence of normalized barrier length on $\bar{A}_{r}(X=1.25, D=1.25)$

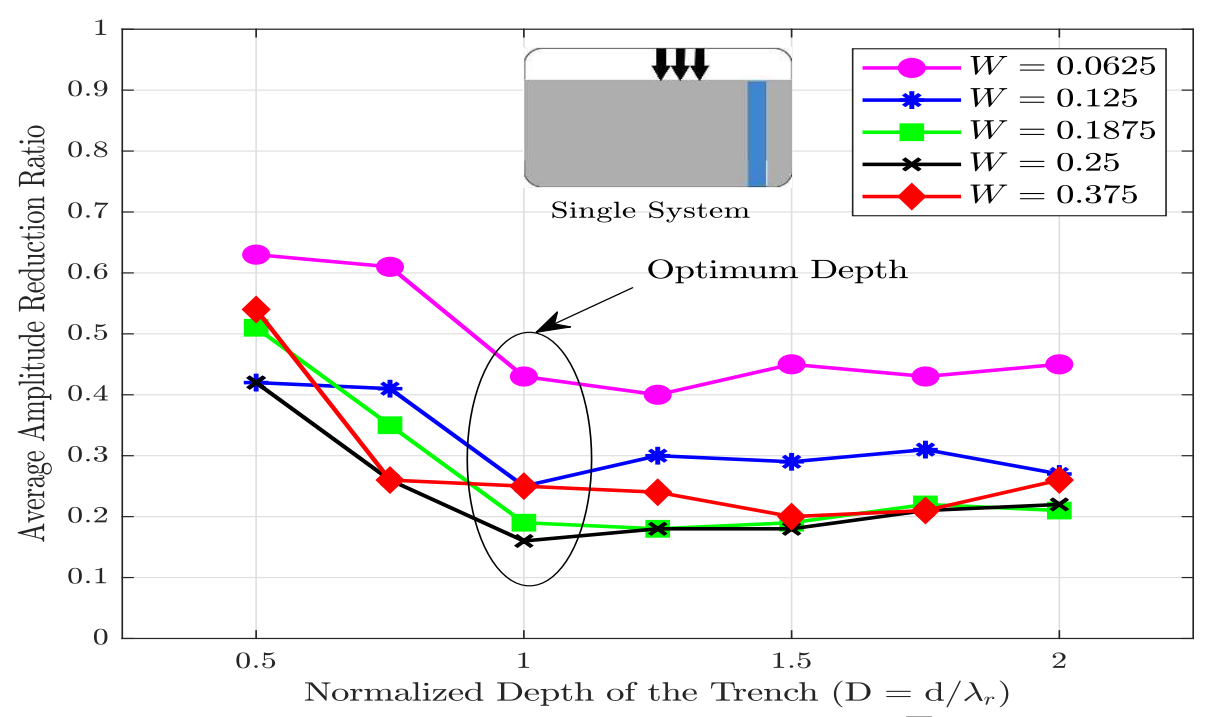

Figure 12: Influence of normalized barrier depth on $\bar{A}_{r}(X=0.5)$ 
there are not significant discrepancies after this depth. Hence, the depth of 1 is defined as the optimum depth for all widths for blocking a major portion of incoming waves. The optimum $D$ has a direct relationship with the width of the barrier.

Fig. 13 shows that $W$ plays a significant role in screening efficiency of the geofoam-filled obstacle. It is apparent that in general the wave attenuation reaches its maximum amount at a $W$ value around 0.25 . For wider trenches $(W>0.2)$, the effect of $W$ is negligible and can be ignored. Increasing the width of the barrier means having more distance between the two sides of the obstacles, which is leading to additional geometric discontinuities in the boundary area and results in a further refraction phenomena of the incoming $\mathrm{R}$-waves.

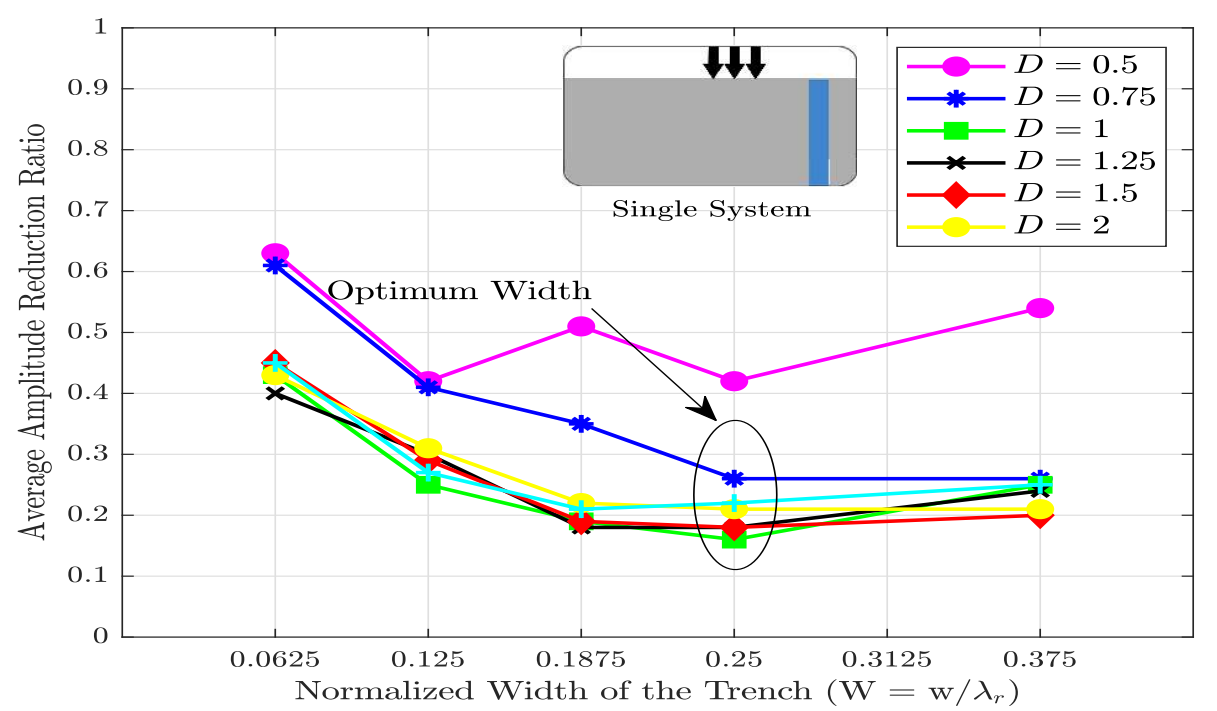

Figure 13: Influence of normalized barrier width on $\bar{A}_{r}(X=0.5)$

\subsection{Double Wall System}

Attenuation of incident waves through a double wall system may be advantageous in circumstances where the implementation of a single wall system is difficult because of unrealistic depth. Using a pair of geofoam-filled barriers may possibly require lower depth in comparison with the single wall system. The efficiency of the double wall system is compared to a single wall with a thickness of $2 W$, having the same width, depth and length as the single wall obstacle. Therefore, the width of both walls are considered as one width, which is the normalized width $W$ for both trenches. At the first step, a parametric study is investigated for finding the optimum spacing between the barriers to achieve the best performance. A parametric study has been done to find the optimum space between the wall and the normalized space of 0.5 is selected for this parametric study. However, the result of the parametric study is not presented in this paper. This space is verified with the results of 31 for optimum distance between trenches. 


\subsubsection{Effect of Trench Dimensions}

The information of Fig. 14 shows that $D$ governs the wave screening for the normalized depth between 0.5 and 1.25 for all widths. The optimum depth of almost 1.25 is selected for all barriers. The results show that $D$ plays a more significant role for narrow trenches than for wider trenches.

At the corners of the trench, mode conversion, that is converting R-wave to body wave occur, due to passing the incoming waves through the edges of the obstacle into the region behind this. Since the diffraction effect is low for a narrow trench $(W=0.0625)$, due to the shorter distance between the two corners of an obstacle, the effect of $D$ is more visible. For wider trenches $(W>0.0625)$, the influence of the diffraction phenomenon is greater than the effect of $D$. All of these issues indicate that the diffraction phenomenon plays an important role in the wave attenuation process for the double wall system.

As seen in Fig 15, $\bar{A}_{r}$ decreases dramatically by increasing the normalized

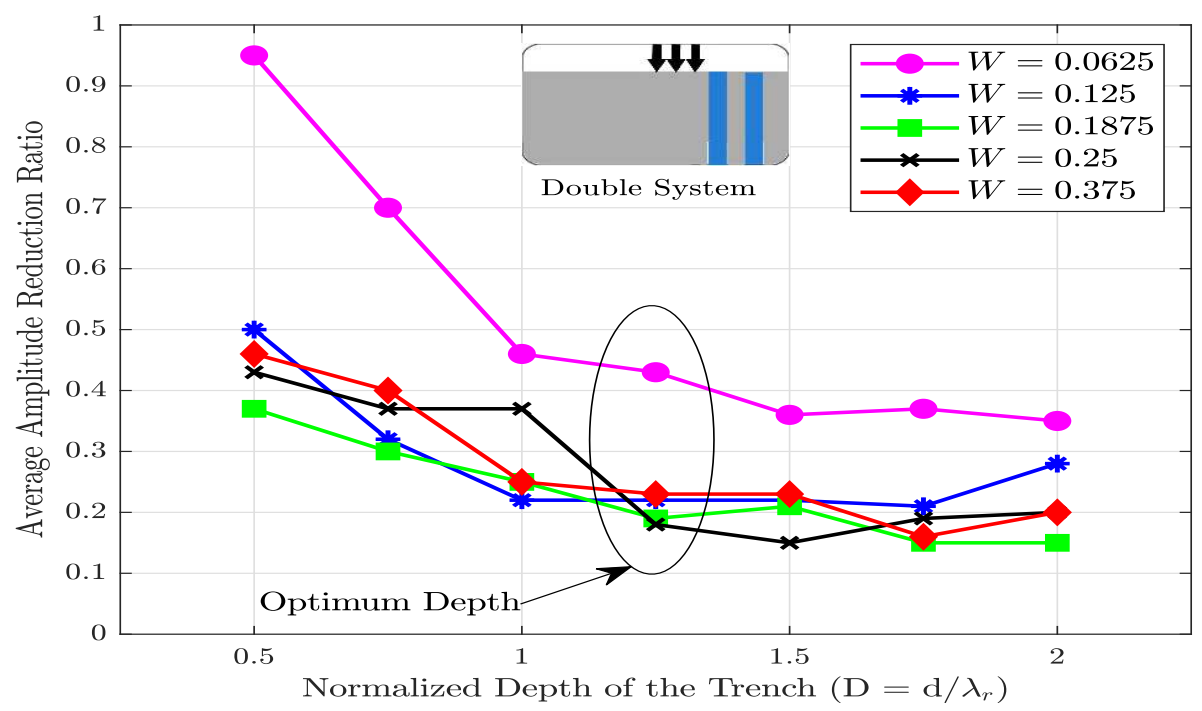

Figure 14: Influence of normalized barrier depth on $\bar{A}_{r}(X=0.5)$

width of the trench from 0.0625 to around 0.2 . Thereafter, it remains at a constant value while $W$ reaches to 0.375 . An optimum width of almost 0.2 is selected for achieving the efficiency of approximately $70 \%$ and $80 \%$. In addition, the influence of $W$ is lower for barriers with $D$ higher than 0.75 and the graphs of deeper trenches follow lower slopes for reaching the optimum width (0.2) from the start point $(W=0.0625)$.

According to developed theory by [42, the energy of R-wave in a region between the ground surface and the R-wavelength (at normalized depth of the trench) is partially reflected at the zone of wave shadow. The R-wave energy passing beneath the obstacle creates a new $\mathrm{R}$-wave at some distance beyond the barrier. The energy of the passing R-wave creates vibrations in the crucial zone. Increasing the width of the trench blocks more incoming waves and lead to passage fewer incident waves. 


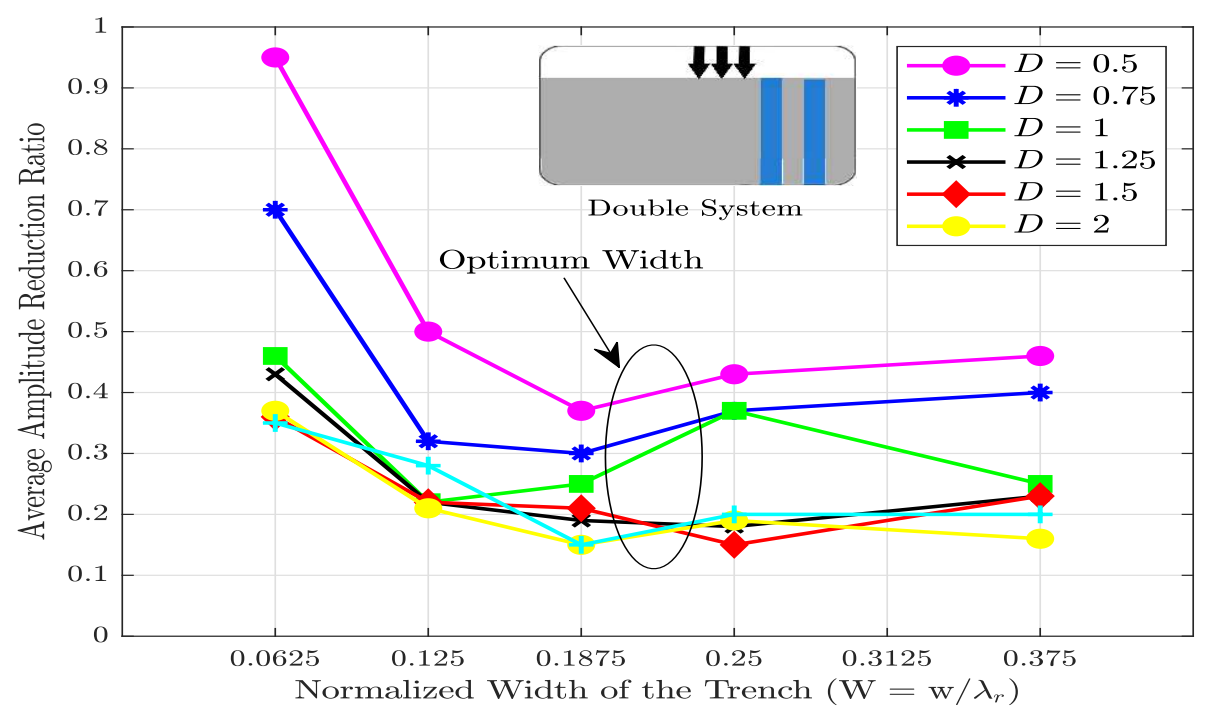

Figure 15: Influence of normalized barrier width on $\bar{A}_{r}(X=0.5)$

\subsection{Triangular Wall System}

A large part of the energy of a dispatched wave is in the form of R-wave. In addition, the amount of transferred energy by incident waves from an interface to the screened zone is a function of the incident angle. The ratio of the reflected to transferred R-wave energy is a function of the corner angle of the trench. The angle of the barrier wall and the ground surface is recognized as one of the most important factors in distributing energy among the waves. Moreover, as discussed in the previous sections for the single and double wall systems, the normalized width of the barrier appears to be one of the most important parameters that will govern the screening effectiveness of the trench. We achieve more wave attenuation by increasing the width of the trench even in the case of shallow trenches. For a proper comparison between all configurations, the triangular system is considered with the same depth and the same cross-sectional area as the single and double wall trench; however, the width of its trench is different from that of the other two systems.

\subsubsection{Effect of Trench Dimensions}

As shown in Fig. 16, increasing the normalized depth of the trench from 0.5 to almost 1.25 for the narrow trench leads to an increase in the efficiency of the trench. However, if the width of the trench increases $(W>0.25)$, a smaller decrease in $\bar{A}_{r}$ is visible in comparison with narrow trenches. A normalized depth of 1 and 1.25 is considered for optimum depth to achieve an acceptable amount of wave attenuation for narrow and wider trenches, respectively. 


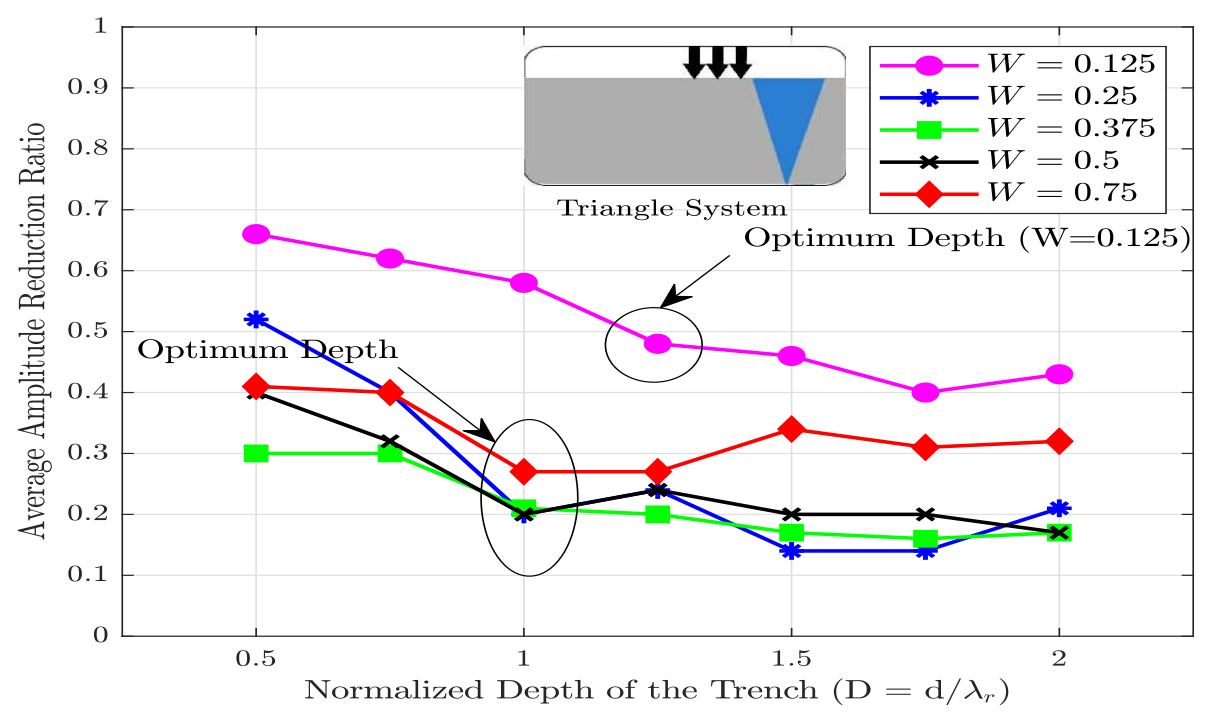

Figure 16: Influence of normalized barrier depth on $\bar{A}_{r}(X=0.5)$

Fig. 17 shows the influence of $W$ on screening performance. A wider trench is sufficient (for $D<0.75$ ) for reaching an acceptable level of screening performance (around $75 \%$ ), while by increasing the normalized depth of the trench $(D>0.75)$, a slender barrier is needed for the effective trench $(W=0.25)$. Increasing the depth of the trench means hindering more incoming waves. For the shallow trench, as the corner of the barrier is near to the ground surface, the role of mode conversion of R-waves into body waves becomes apparent. However, in the case of deeper trenches, the corner of the trench move further into the soil. Therefore, the effect of the diffraction, which is resulting of passing the wave around a trench edge (trench corner) becomes smaller. Thus, a slender obstacle is needed to achieve an admissible amount of vibration for deep trenches.

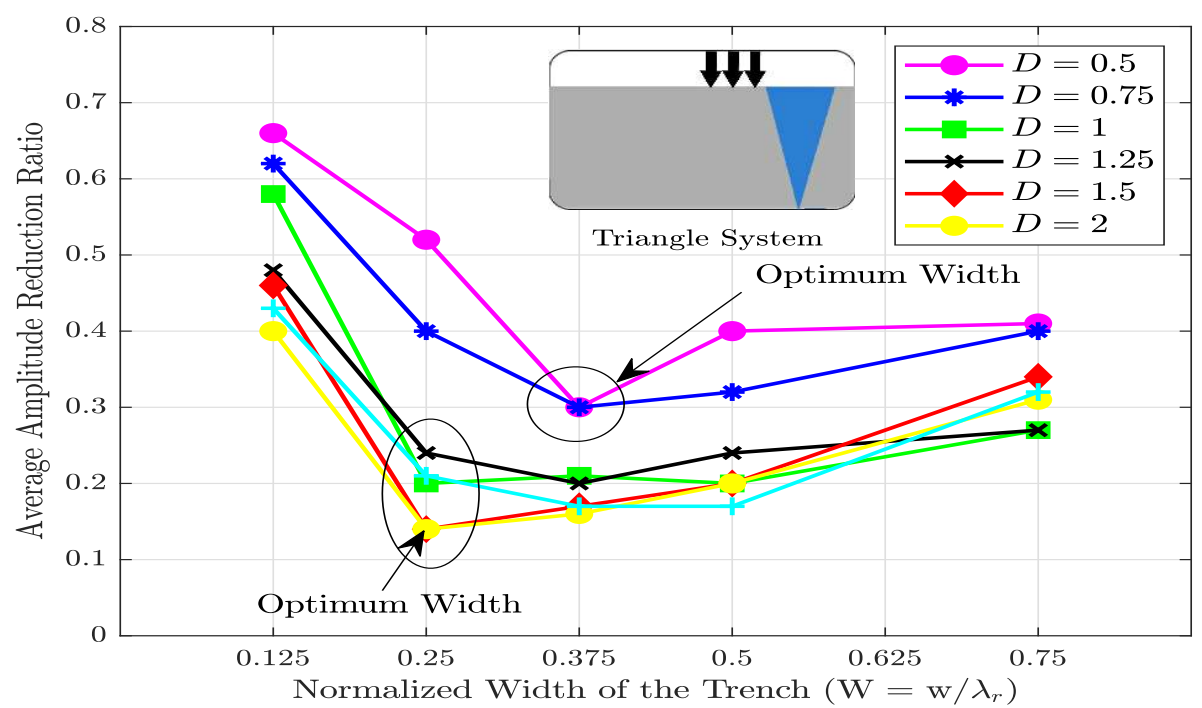

Figure 17: Influence of normalized barrier width on $\bar{A}_{r}$ of EPS Geofoam wave barrier $(X=0.5)$ 


\section{Passive Isolation}

A $2 D$ time domain finite element model is established for presenting the result of the parametric investigation. Increasing the normalized distance between the obstacle and vibration source can lead to making $\mathrm{R}$-waves more prevailing in comparison with body waves. 3, 18. If the barrier encounters plane waves, a plane strain model can present reliable results for calculating average amplitude reduction ratio. Another reason is saving the computational time. Since the geometry of passive is bigger than active model, analysing several models would require a lot of time. In addition, several researches [11, 26] showed that the result of $2 D$ and $3 D$ models are in acceptable agreement. Different parameters including trench depth, width and location are considered for studying the effectiveness of the trench.

\subsection{Single Wall Barrier}

The variation of the barrier's dimensional factors (including depth and width) is determined in the same way as in the active isolation scheme. However, the geometric factor (including distance between the obstacle and vibration source) is determined to be 3 . The reduction zone study of the single wall trench, which is not presented in detail, showed a considerable reduction in vertical ground displacement before and after installing the geofoam-filled trench for a normalized distance of 10 , which is selected for calculating $\bar{A}_{r}$.

\subsubsection{Geometric Factor Study}

The result of $\bar{A}_{r}$ as a function of $W$ is plotted in Fig. 18. A careful study of this figure reveals that there is no substantial relationship between the computed $\bar{A}_{r}$ value and the normalized depth. On the other hand, increasing the wall

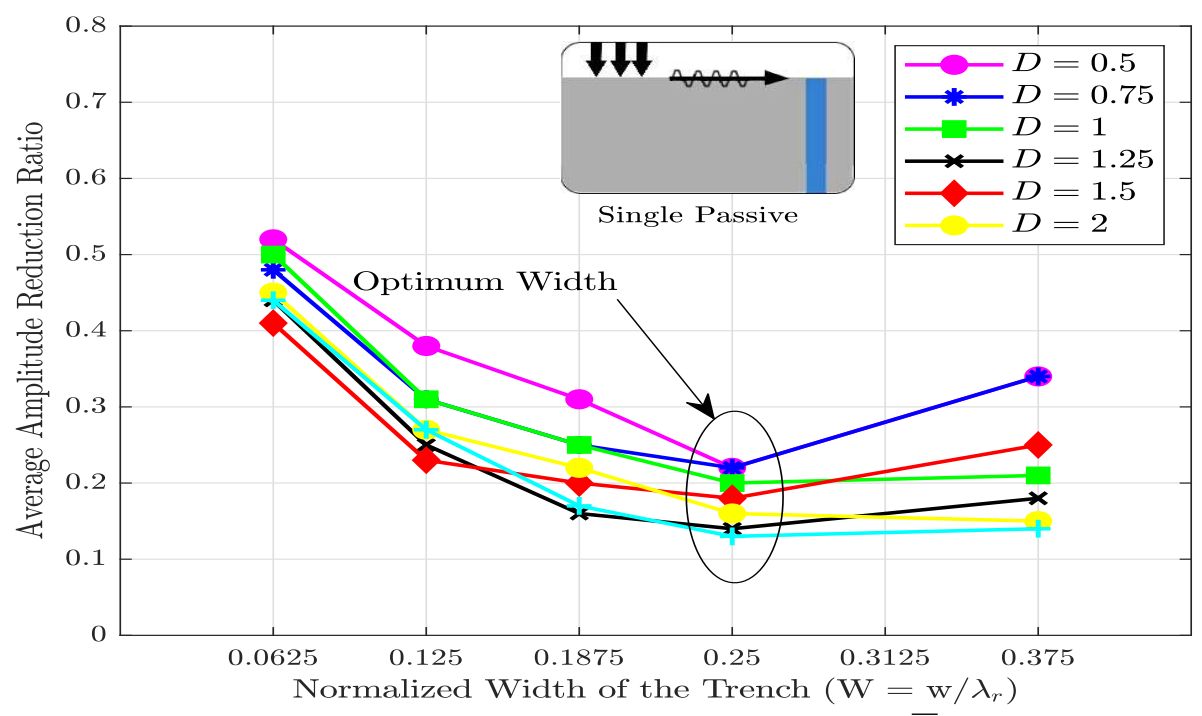

Figure 18: Influence of normalized barrier width on $\bar{A}_{r}(X=3)$

thickness leads to further scattering of the ground vibrations generated by source of disturbance. A reduction of approximately $60 \%$ is achieved by increasing $W$ 
from 0.0625 to 0.25 for almost all trench depths and it seems that $W=0.25$ is the optimum width for achieving a reasonable amount of wave attenuation. An increase in trench thickness creates more discontinuity at the ground surface with different dynamic properties and this difference creates a diffraction in incident waves.

\subsection{Double Wall Barrier}

Fig. 19 shows that $\bar{A}_{r}$ is not affected by the trench depth and even increasing $W$ cannot create any change in the influence of $D$ on $\bar{A}_{r}$. Some of the incident $\mathrm{R}$-waves reflect downward to the left of the obstacle after encountering the left side of first wall and the remaining one transmit to the right of the barrier. Subsequently, the transmitted R-waves reflect again through the discontinuity at the second wall. In fact, the diffraction and mode conversation due to the corners of a pair of trenches play more significant role in decaying the incoming R-waves in comparison with the depth, and this issue outweighs the role of trench depth.

Wider barriers lead to better screening effect and $W$ seems to govern the screening process. For the narrow trench however, increasing the normalized width $(W>0.2)$ results in decreasing the effect of $W$, owning the fact that increasing the width of the barriers hardly affects the screening vibration at any depth. Since the thickness of the obstacle reaches a certain value, increasing width of the barrier hardly affect the screening vibration for almost all depths. Increasing the normalized width leads to more discontinuity in the ground surface with different shear modulus hindering more ground-born vibration. However, since $W$ continuously increases, the probability of having wave interferences among the R-waves that are propagated through the trench and the waves that are transmitted beneath the obstacle due to different velocity. This phenomenon may be one of the reasons for reaching to a stable value of the normalized width beyond 0.25 .

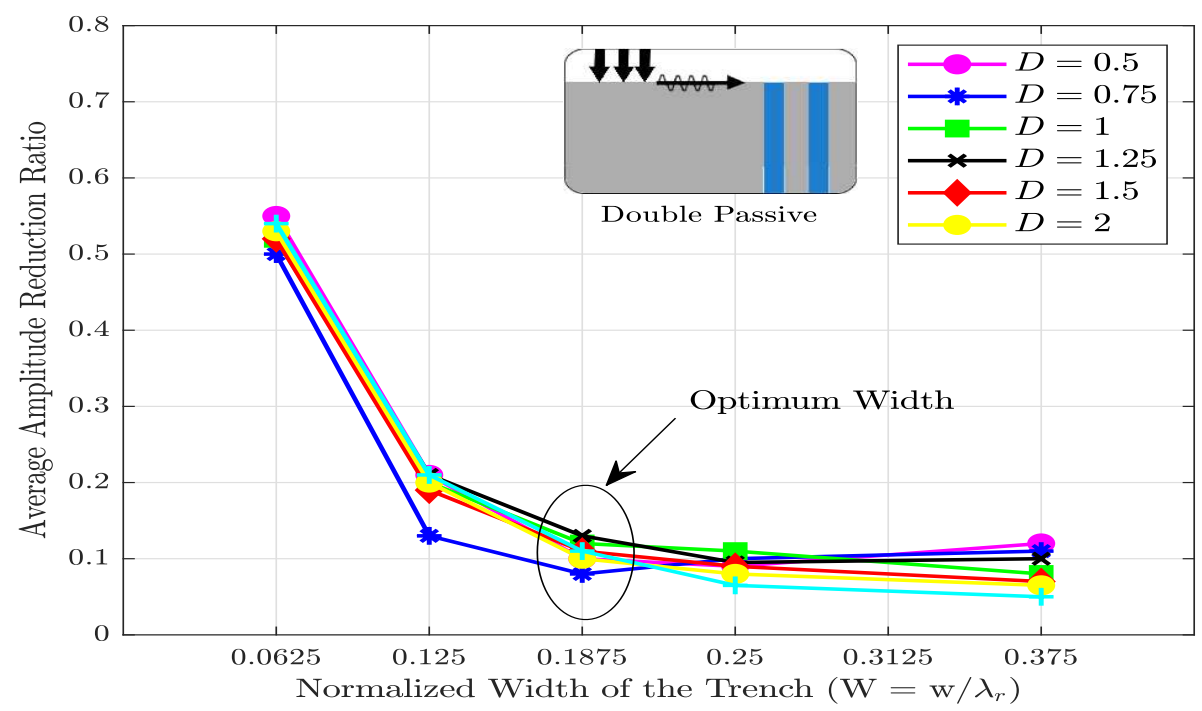

Figure 19: Influence of normalized barrier width on $\bar{A}_{r}(X=3)$ 


\subsection{Triangular Wall Barrier}

As it can be seen in Fig. 20, the efficiency of the trench increases gradually and continuously by increasing the normalized depth for approximately all widths, implying that $D$ governs the scattering influence. It is apparent from the figure that the barrier width has an ignorable influence on the scattering of incoming energy. Considering the corner of the triangular wall barrier, its shape can be the reason for observing no mode conversion or diffraction phenomenon that changes the R-waves to body waves.

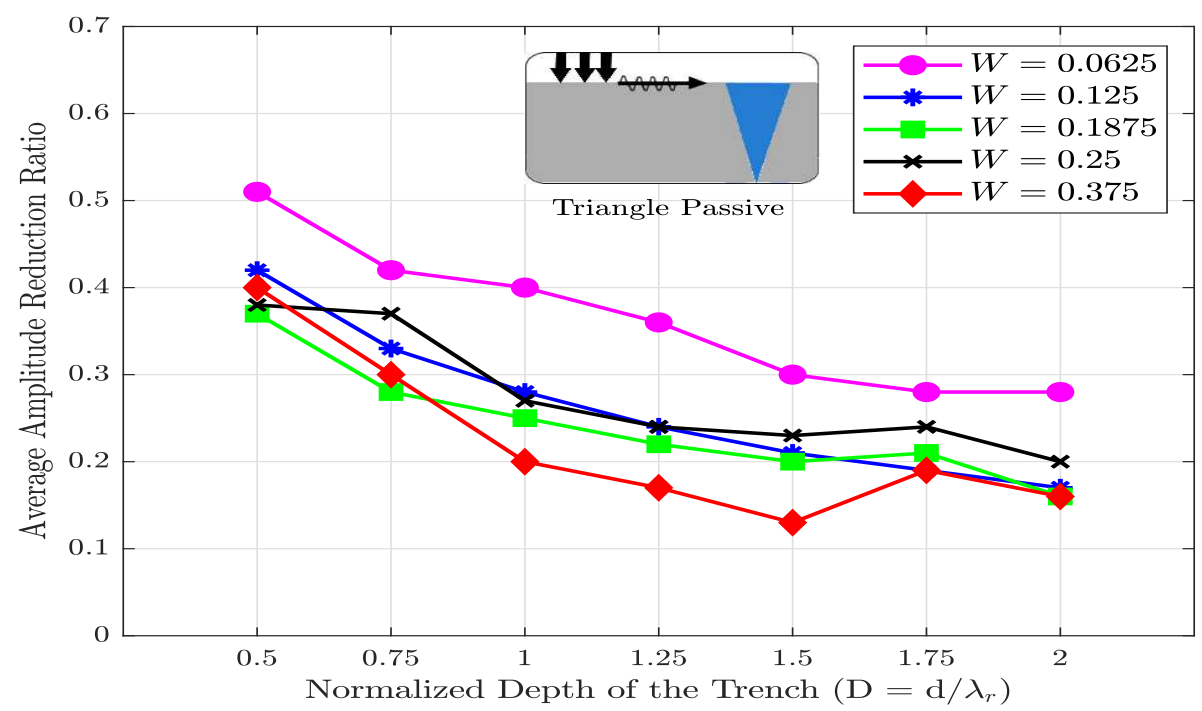

Figure 20: Influence of normalized barrier depth on $\bar{A}_{r}(X=3)$

\section{Conclusion}

Based on a fully experimental test, a comprehensive numerical modelling for evaluating the efficiency of the geofoam-filled barrier in attenuating dispatched waves has been performed. The conclusions drawn from field work and parametric studies can be summarized as follows:

Active isolation can be a better solution for hindering of incident waves in comparison with the passive isolation based on the results of field test. However, the results also showed that the first point after the trench has the highest amount of mitigation and normalized depth of $0.6 \lambda_{\mathrm{r}}$ is enough for reaching the highest value of the efficiency for both active and passive isolation systems.

The parametric study of the active and passive isolations showed the zone of $7 \lambda_{\mathrm{r}}$ and $10 \lambda_{\mathrm{r}}$ as crucial zones for an area after the trench for computing $\bar{A}_{r}$. This finding proves that the vibration isolation system should be divided into active and passive systems during analysing.

Analysing the result of the active scheme demonstrated that the optimum length can be considered as $2.5 \lambda_{\mathrm{r}}$ for all three systems for reaching the highest value of the efficiency. The depths of $1 \lambda_{\mathrm{r}}, 1.2 \lambda_{\mathrm{r}}$ and $1 \lambda_{\mathrm{r}}$ are considered as the optimum depths for the single, double and triangular wall systems, respectively. Concerning the effect of width of the geofoam-filled trench, it is concluded that the value of $W=0.2,0.2$ and 0.25 are enough for considering the optimum $W$ 
for single, double and triangular wall systems, respectively.

On the other hand, evaluation of the passive isolation revealed that the depth of the barrier hardly affects the efficiency of the obstacle for single and double wall systems and the influence of $D$ can be ignored as long as $D>0.5$. While, $D$ is a key factor in scattering of incoming waves for the triangular wall system. However, an optimum depth cannot be selected for this system as the efficiency of the obstacle increases gradually and continuously by increasing $D$. The normalized width has to be increased to approximately 0.25 to have a successful single wall system. For the double wall system, $W$ should be at least 0.2 for having satisfactory influence of screening. The barrier's width has a negligible effect on the scattering of dispatched waves for the triangular wall system. In addition, the parametric study of the trench location for both active and passive schemes demonstrated that this factor is not an important parameter that influences the efficiency of the geofoam-filled trench.

\section{References}

[1] GF Miller, H Pursey, and Edward Crisp Bullard. On the partition of energy between elastic waves in a semi-infinite solid. Proceedings of the Royal Society of London. Series A. Mathematical and Physical Sciences, 233(1192):55-69, 1955.

[2] Frank Edwin Richart, John Russell Hall, and Richard D Woods. Vibrations of soils and foundations. 1970.

[3] Tahmeed Malik Al-Hussaini. Vibration isolation by wave barriers. 1993.

[4] KR MASSARSCH. Vibration isolation using gas-filled cushions, soil dynamics symposium to honor prof. richard d. woods. Geofrontiers 2005, Austin, Texas, January 24-26, 2005.

[5] E Mahdavisefat, H Salehzadeh, and AA Heshmati. Full-scale experimental study on screening effectiveness of srm-filled trench barriers. Géotechnique, 68(10):869-882, 2018.

[6] Pieter Coulier, Vicente Cuéllar, Geert Degrande, and Geert Lombaert. Experimental and numerical evaluation of the effectiveness of a stiff wave barrier in the soil. Soil Dynamics and Earthquake Engineering, 77:238-253, 2015.

[7] Guangyun Gao, Qiwei Zhang, Juan Chen, and Qingsheng Chen. Field experiments and numerical analysis on the ground vibration isolation of wave impeding block under horizontal and rocking coupled excitations. Soil Dynamics and Earthquake Engineering, 115:507-512, 2018.

[8] Tulika Bose, Deepankar Choudhury, Julian Sprengel, and Martin Ziegler. Efficiency of open and infill trenches in mitigating ground-borne vibrations. Journal of Geotechnical and Geoenvironmental Engineering, 144(8):04018048, 2018.

[9] Richard D Woods. Screening of suface waves in soils. 1968. 
[10] D Ulgen and O Toygar. Screening effectiveness of open and in-filled wave barriers: a full-scale experimental study. Construction and Building Materials, 86:12-20, 2015.

[11] Ashref Alzawi and M Hesham El Naggar. Full scale experimental study on vibration scattering using open and in-filled (geofoam) wave barriers. Soil Dynamics and Earthquake Engineering, 31(3):306-317, 2011.

[12] Xingbo Pu, Zhifei Shi, and Hongjun Xiang. Feasibility of ambient vibration screening by periodic geofoam-filled trenches. Soil Dynamics and Earthquake Engineering, 104:228-235, 2018.

[13] Erkan Çelebi, Seyhan Fırat, Günay Beyhan, İlyas Çankaya, İsa Vural, and Osman Kirtel. Field experiments on wave propagation and vibration isolation by using wave barriers. Soil Dynamics and Earthquake Engineering, 29(5):824-833, 2009.

[14] Carol Murillo, Luc Thorel, and B Caicedo. Ground vibration isolation with geofoam barriers: Centrifuge modeling. Geotextiles and Geomembranes, 27(6):423-434, 2009.

[15] DE Beskos, B Dasgupta, and IG Vardoulakis. Vibration isolation using open or filled trenches. Computational mechanics, 1(1):43-63, 1986.

[16] KL Leung, DE Beskos, and IG Vardoulakis. Vibration isolation using open or filled trenches. Computational Mechanics, 7(2):137-148, 1990.

[17] Dimitri E Beskos. Boundary element methods in dynamic analysis: Part ii (1986-1996). Applied mechanics reviews, 50(3):149-197, 1997.

[18] S Ahmad and TM Al-Hussaini. Simplified design for vibration screening by open and in-filled trenches. Journal of geotechnical engineering, 117(1):67$88,1991$.

[19] WA Haupt. Wave propagation in the ground and isolation measures. 1995.

[20] WA Haupt. Isolation of vibrations by concrete core walls. In Proceedings of the ninth international conference on soil mechanics and foundation engineering, volume 2, pages 251-256, 1977.

[21] Sanka D Ekanayake, DS Liyanapathirana, and Chin Jian Leo. Attenuation of ground vibrations using in-filled wave barriers. Soil Dynamics and Earthquake Engineering, 67:290-300, 2014.

[22] DS Liyanapathirana and Sanka D Ekanayake. Application of eps geofoam in attenuating ground vibrations during vibratory pile driving. Geotextiles and Geomembranes, 44(1):59-69, 2016.

[23] Mainak Majumder, Priyanka Ghosh, and S Rajesh. An innovative vibration barrier by intermittent geofoam-a numerical study. Geomechanics and Engineering, 13(2):269-84, 2017.

[24] Mainak Majumder, Priyanka Ghosh, and S Rajesh. Numerical study on intermittent geofoam in-filled trench as vibration barrier considering soil nonlinearity and circular dynamic source. International Journal of Geotechnical Engineering, 11(3):278-288, 2017. 
[25] Mainak Majumder and Priyanka Ghosh. Intermittent geofoam in-filled trench for vibration screening considering soil non-linearity. KSCE Journal of Civil Engineering, 20(6):2308-2318, 2016.

[26] Qiu Bo, Limam Ali, and Djeran-Maigre Irini. Numerical study of wave barrier and its optimization design. Finite Elements in Analysis and Design, 84:1-13, 2014

[27] Ankurjyoti Saikia. Numerical study on screening of surface waves using a pair of softer backfilled trenches. Soil Dynamics and Earthquake Engineering, 65:206-213, 2014.

[28] M Schevenels, G Lombaert, et al. Double wall barriers for the reduction of ground vibration transmission. Soil Dynamics and Earthquake Engineering, 97:1-13, 2017.

[29] Morteza Esmaeili, Jabbar Ali Zakeri, and Seyed Ali Mosayebi. Investigating the optimized open v-shaped trench performance in reduction of train-induced ground vibrations. International Journal of Geomechanics, 14(3):04014004, 2013.

[30] Jabbar-Ali Zakeri, Morteza Esmaeili, and Seyed-Ali Mosayebi. Numerical investigation of the effectiveness of a step-shaped trench in reducing traininduced vibrations. Proceedings of the Institution of Mechanical Engineers, Part F: Journal of Rail and Rapid Transit, 228(3):298-306, 2014.

[31] Ashref Alzawi, M Hesham El Naggar, and Ph DP Eng. Numerical investigations on vibration screening by in-filled geofoam trenches. In $64 \mathrm{rd}$ Canadian Geotechnical Conference, Toronto, ON, Canada,(Accepted), 2011.

[32] Julian Sprengel. Optimierung der praktischen Anwendbarkeit aufgeschäumter Isolierkörper im Boden zur Erschütterungsreduktion. PhD thesis, RWTH Aachen University, 2017.

[33] D. Heiland M. Mistler. Mobiler shaker für die künstliche ersatzanregung bei baudynamischen fragestellungen. Messtechnik im Bauwesen, 2014.

[34] Ankurjyoti Saikia. Numerical investigation on vibration isolation by softer in-filled trench barriers. Journal of Geo-Engineering Sciences, 3(1):31-42, 2016.

[35] GA Athanasopoulos, PC Pelekis, and GA Anagnostopoulos. Effect of soil stiffness in the attenuation of rayleigh-wave motions from field measurements. Soil Dynamics and Earthquake Engineering, 19(4):277-288, 2000.

[36] DK Baidya and G Murali Krishna. Investigation of resonant frequency and amplitude of vibrating footing resting on a layered soil system. Geotechnical Testing Journal, 24(4):409-417, 2001.

[37] Roger L Kuhlemeyer and John Lysmer. Finite element method accuracy for wave propagation problems. Journal of Soil Mechanics 83 Foundations Div, 99(Tech Rpt), 1973. 
[38] SE Kattis, D Polyzos, and DE Beskos. Modelling of pile wave barriers by effective trenches and their screening effectiveness. Soil Dynamics and Earthquake Engineering, 18(1):1-10, 1999.

[39] Izzat Muhammad Idriss and Harry Bolton Seed. The response of earth banks during earthquakes. Technical report, 1966.

[40] M Iodice, E Rustighi, and J Muggleton. Comparison of soil excitation methods for surface wave speed measurements. 2014.

[41] Edward L Wilson. Three-dimensional static and dynamic analysis of structures. 2002.

[42] Hans-Joachim Dolling. Abschirming von erschütterungen durch bodenschlitze. Die Bautechnik, 5:151-158, 1970. 